\title{
Sinus Disorder, CTCAE
}

National Cancer Institute

\section{Source}

National Cancer Institute. Sinus Disorder, CT CAE. NCI Thesaurus. Code C143831.

A disorder characterized by involvement of the paranasal sinuses. 